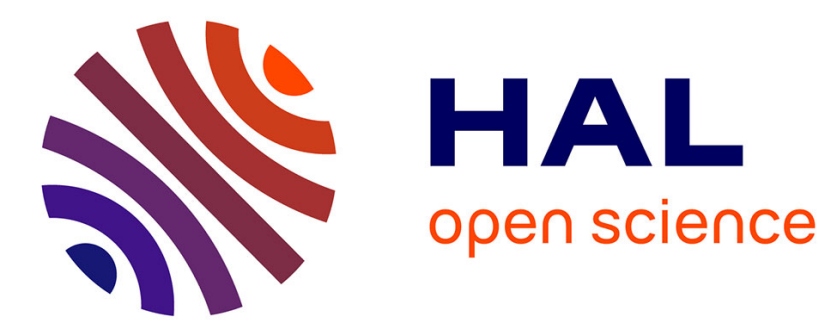

\title{
Some aspects of Lagrangian dynamics of turbulence
}

Mickaël Bourgoin

\section{To cite this version:}

Mickaël Bourgoin. Some aspects of Lagrangian dynamics of turbulence. Mixing and dispersion in flows dominated by rotation and buoyancy, 2018. hal-02633932

\section{HAL Id: hal-02633932 \\ https://hal.science/hal-02633932}

Submitted on 27 May 2020

HAL is a multi-disciplinary open access archive for the deposit and dissemination of scientific research documents, whether they are published or not. The documents may come from teaching and research institutions in France or abroad, or from public or private research centers.
L'archive ouverte pluridisciplinaire HAL, est destinée au dépôt et à la diffusion de documents scientifiques de niveau recherche, publiés ou non, émanant des établissements d'enseignement et de recherche français ou étrangers, des laboratoires publics ou privés. 


\title{
Some aspects of Lagrangian dynamics of turbulence
}

\author{
Mickaël Bourgoin \\ Univ Lyon, Ens de Lyon, Univ Claude Bernard, CNRS, Laboratoire de Physique, F-69342 Lyon, \\ France
}

\section{Contents}

Contents

1 Introduction

2 Basic multi-scale properties of Lagrangian turbulence 3

2.1 A brief recall of K41 ideas in the Eulerian framwork . . . . . . . . . . . . . . . . 3

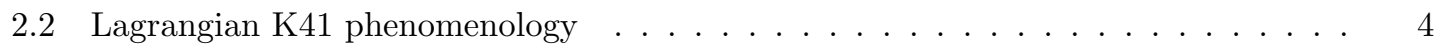

2.3 Lagrangian dispersion . . . . . . . . . . . . . . . . . . . 5

3 On the validity of Kolmogorov's local isotropy hypothesis 6

3.1 The local isotropy hypothesis . . . . . . . . . . . . . . . . . . 6

3.2 The local isotropy hypothesis in large scale anisotropic flows . . . . . . . . . . . 7

3.3 The influence of increasing Reynolds number . . . . . . . . . . . . . . . 8

4 Turbulent dispersion of tracer particles $\quad 9$

4.1 The turbulent pair dispersion problem . . . . . . . . . . . . . . . . . . . . 9

4.2 Batchelor and Richardson regimes for pair dispersion . . . . . . . . . . . . . . . 10

4.3 A simple ballistic phenomenology of turbulent superdiffusion . . . . . . . . . . . 13

4.4 Practical implementation of the ballistic phenomenology . . . . . . . . . . . 15

4.5 About the time irreversibility of turbulent relative dispersion . . . . . . . . . . 16

$\begin{array}{llr}5 & \text { Conclusion } & 18\end{array}$

Bibliography

Abstract This chapter is dedicated to fundamental properties of Lagrangian transport in turbulence, emphasizing the role of of anisotropy and 2D turbulence which are relevant for geophysical considerations. The focus is on three main aspects of Lagrangian turbulence : (i) the role of small scale anisotropy on the Lagrangian energy spectrum, (ii) the role of Lagrangian intermittency and (iii) the relative dispersion of tracer particles.

\section{Introduction}

Turbulence governs the vast majority of fluid flows in nature and industrial applications from atmospheric dynamics to mixing and combustion. Despite its ubiquitousness, turbulence remains one of the deepest unsolved mysteries of classical physics. Even if the equations of motion of turbulent flows are perfectly known since Navier and Stokes, almost two centuries ago, its complexity (primarily driven by its non-linearity) annihilates any hope of finding analytical solutions. We are then committed to turn to phenomenological modeling to gain insight into the behavior of turbulent flows. In 1922 L. F. Richardson introduced the first description of turbulence as a multiple scale phenomenon (Richardson cascade) where mechanical energy is injected at large eddies (with typical scale $L$, referred to as injection or integral scale), and as they become unstable, they split into smaller eddies to which energy is transferred, and so on until eventually viscous dissipation stops the cascade at some small scale ( $\eta$, referred to as dissipative scale) where viscosity becomes 
dominant. This range of scales defines the inertial range of turbulence. In turbulent flows, the range of inertial range between the energy injection scale $L$ and the dissipative scale $\eta$ is directly related to the Reynolds number $R e$ of the flow: $L / \eta \propto R e^{3 / 4}$ (see for instance Tennekes (1975)). Reynolds numbers of the order $10^{6}$ are usual in geophysical flows, implying that at least 4 decades of spatial dynamics are typically involved. Similarly in the time domain, the ratio between the eddy turnover time $T$ of eddies at injection scale and the eddy turnover time $\tau_{\eta}$ of eddies at dissipation scale goes as $T / \tau_{\eta} \propto R e^{1 / 2}$, covering typically three decades of temporal dynamics. These dynamical ranges can be even further extended toward the largest scales due to inverse cascade mechanisms, which may become important in the atmosphere or the ocean, at scales where dynamics exhibits 2D-properties, where flow structures can extend over hundreds of kilometers. The notion of energy cascade is therefore at the core of the physics of turbulence and its multi-scale nature. We know today that this cascade results from the non-linear interaction of Fourier modes of the velocity field, although we are still unable to analytically model and predict most of turbulence statistics over the entire range of inertial scales. In $1941 \mathrm{~A}$. Kolmogorov proposed a quantitative statistical description of turbulence (Kolmogorov (1941); Frisch (1995)) as a self-similar cascade with universal properties. Originally this universality was stated by two self-similar hypotheses which are the foundation of Kolmogorov's phenomenology. Quoting Hinze's formulation (Hinze (1959)), these hypothesis state that:

H1. At sufficiently high Reynolds number there is a range of small-scales for which turbulence is statistically in equilibrium and uniquely determined by the viscosity $\nu$ and the mean energy dissipation rate $\langle\epsilon\rangle$; this equilibrium state is universal ;

H2. In the limit of infinite Reynolds number turbulence become independent of viscosity $\nu$ and solely determined by $\langle\epsilon\rangle$.

Taken together, Kolmogorov's hypotheses state that in intense turbulence and well away from any boundaries or singularities the statistics of turbulent flow should be universal at length and timescales that are small compared with the injection of energy into the flow. This implies in particular that at sufficiently small scales, turbulence becomes homogeneous and isotropic, an hypothesis known as local isotropy. In Kolmogorov's approach the mean energy dissipation rate $\langle\epsilon\rangle$ then becomes the only relevant parameter governing the dynamics of structures in the inertial range (for clarity, in the sequel we shall refer to $\langle\epsilon\rangle$ simply as $\epsilon$ ). Since then, statistical turbulence modeling has been dominated by Kolmogorov's ideas, whose 1941 hypotheses have so influenced the field that they are simply known as the K41 phenomenology. The great utility of the K41 model lies in its prediction of universal scaling laws for velocity increments statistics. However, K41 is known to fail describing important features of real turbulent flows, as for instance the intermittency phenomenon. This has stimulated many theoretical studies trying to refine K41 phenomenology, including Kolmogorov's refined hypotheses of self-similarity in 1962 (known as K62) and more recently multifractal models (Parisi and Frisch (1985)).

In the same spirit of finding alternative, or at least complementary, descriptions of turbulence, new modeling paths have emerged, among which the Lagrangian approach offers promising opportunities. Fluid dynamics in general, and turbulence in particular, is usually described in a Eulerian approach: the fluid velocity (or any other physical quantity as pressure, fluid acceleration, etc.) is studied as a spatial field $\vec{v}_{E}(\vec{r})$, function of the spatial coordinate $\vec{r}$. Although this field also experiences instantaneous temporal fluctuations (and should be written $\vec{v}_{E}(\vec{r}, t)$ ), in statistically stationary conditions, time $t$ is generally considered as a statistical parameter which simply helps building ensemble averages by repeating the measurement at different instants (if the system is not statistically stationary, then time $t$ becomes an actual variable of the problem which accounts for non-stationary effects). Eulerian statistics are typically acquired by measuring the velocity (or any other quantity) at fixed points where different fluid particles are being probed at every instant. In the Lagrangian approach, instead of probing the flow at given fixed points $\vec{r}$ (where fluid particles do stream continuously), velocity is measured along the path of given fluid elements which are tagged and tracked individually (see fig. 1). Whereas most of our knowledge of turbulence (including Kolmogorov's ideas) comes from the Eulerian approach, the Lagrangian approach offers complementary observations. Moreover in several cases the Lagrangian point of view offers 


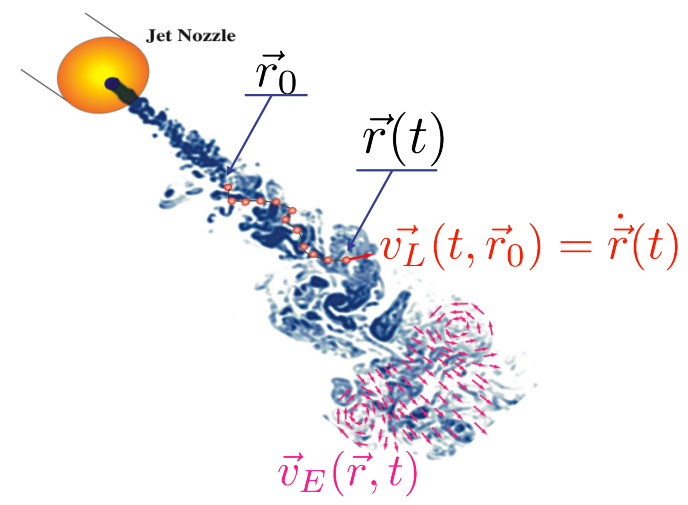

Figure 1. Eulerian versus Lagrangian description of a flow. In the Eulerian framework the flow is described in terms of the velocity field, while in the Lagrangian framework it is characterized from the trajectories of fluid tracers.

a more natural framework Yeung (2002). This is the case for instance for dispersion issues (and related mixing problems) for which a particulate description (rather than a field approach) is often more relevant. As a matter of fact, in the course of its long history, the study of turbulent flows always benefited from comings and goings between a field view and a particle view, that is between an Eulerian description and a Lagrangian approach. The relevance of Lagrangian approach to address transport properties of turbulence was already pointed the 1920?s in pioneering articles by Taylor (1922) and Richardson (1926). However, the Lagrangian statistical formalism suffered from a lack of reliable and precise experimental measurements. It is only during the last decades that, stimulated by important theoretical breakthroughs (for instance in the context of stochastic Lagrangian models of turbulence (Sawford (1991))), new highly sophisticated laboratory experiments (Bourgoin et al. (2014)) and high-resolution direct numerical simulations have attacked seriously the problem of Lagrangian turbulence, and more widely, the problem of the transport of impurities (not restricted to infinitesimal material fluid elements) by a turbulent flow (Bourgoin and $\mathrm{Xu}(2014))$.

In this context, this chapter briefly recalls some of the main properties of Lagrangian turbulence in homogeneous isotropic turbulence, before addressing two important aspects, possibly relevant for atmospheric and oceanographic flows, which can be impacted by additional complexities such as stratification, global rotation and aspect ratio, related to anisotropy and bi-dimensionalization of turbulence. The role of these additional complexities will be presented from simplified experiments or models.

In this context, the present chapter focuses on two important questions of Lagrangian turbulence

- The validity of Kolmogorov's local isotropy hypothesis ;

- The relative dispersion of pairs ;

\section{Basic multi-scale properties of Lagrangian turbulence}

\subsection{A brief recall of K41 ideas in the Eulerian framwork}

Since Kolmogorov's developments in 1941, the multi-scale nature of turbulence is described by means of statistics of velocity increments. In classical Eulerian approaches, spatial velocity differences are generally considered $\delta_{E} \boldsymbol{u}(r)=\boldsymbol{u}(\boldsymbol{r}+\boldsymbol{x})-\boldsymbol{u}(\boldsymbol{x})$ and statistics are investigated in terms of their moments $S_{p}^{E}(r)=<\delta_{E} \boldsymbol{u}(\boldsymbol{r})^{p}>$ also called structure functions. Such incremental quantities are important for several reasons. First because the classical inertial range similarity hypothesis since K41 is best suited to increments rather than to raw velocity field. With this respect we can quote Batchelor who wrote about the importance of velocity differences in this context, that "it seems reasonable to suppose that when $r$ is small enough the larger eddies make very little contribution to the velocity difference, so that this mean value is determined almost 
wholly by eddies whose diameters are of the same or smaller order than $r$, and we led to apply similarity hypotheses" Batchelor (1950). Increments are also important because they are directly related to common major statistical quantities, particularly in the case of second order structure function $S_{2}(r)=<\delta u(r)^{2}>$ which, for homogeneous turbulence, is directly related to the velocity Eulerian correlation function $R_{u u}^{E}(r)=\sigma_{u}^{2}\left(1-S_{2}^{E}(r) / 2 \sigma_{u}^{2}\right)$ (with $\sigma_{u}^{2}$ the velocity variance) which is itself related to the velocity spectrum by simple Fourier transform. A last important aspect of increments comes from the third order Eulerian structure function for which one of the only known exact analytical results (the celebrated Kolmogorov 4/5th law) is directly derivable from Navier-Stokes equations and states that $S_{3}^{\|}(r)=-\frac{4}{5} \epsilon r$ (where $S_{3}^{\|}(r)$ is the longitudinal third order structure function). In K41 phenomenology, the self-similarity hypothesis imply that the dynamics of turbulence in the inertial range of scales (far from injection scale and far from dissipation), the only physical relevant parameter is the energy dissipation rate $\epsilon$. This has an important implication regarding the scaling of spatial velocity increments, as simple dimensional analysis therefore imply that $\left\langle\delta_{E} \boldsymbol{u}(\boldsymbol{r})^{p}>\propto(\epsilon r)^{p / 3}\right.$. Important results of K41 phenomenology concern the consistency with the aforementioned 4/5th law (for $p=3$ ) and the prediction of the celebrated $\epsilon^{2 / 3} k^{-5 / 3}$ energy spectrum (with $k$ the spatial wave number) is the Fourier space equivalent of the scaling for $S_{2}(r) \propto(\epsilon r)^{2 / 3}$.

Closer studies, however, have shown that the K41 scaling predictions are not obeyed, especially for high-order moments. Instead, the $\zeta_{p}^{E}$ increase nonlinearly and slower than $\mathrm{p}=3$ (Anselmet et al., 1984; Arneodo et al., 1996; Frisch, 1995; Chen et al., 2005). This anomalous scaling is usually attributed to the phenomenon of intermittency that destroys the perfect self-similarity underlying the K41 phenomenology, and reflects the fact that energy dissipation $\epsilon$ is highly unevenly distributed. As an attempt of definition of intermittency (a consensual definition has not emerged yet in the turbulence community) we may see it as the fact that statistical quantities of a turbulent field dependent on the scale at which they are explored. As a consequence, if we consider increments at a given scale $r$ of a given velocity field intermittency causes their probability density function (PDF) to change depending on the observation scale $r$. Such a deformation of the increments PDF is now well-established and well characterized for Eulerian fields (though its physical origin remains mysterious): increments PDF are gaussian at large scale ; they develop approximately exponential tails for separations in the inertial range; tails become stretch exponential at even smaller scales. These evolution of the statistics across the scales reveals the non self-similar nature of turbulence, in contradiction with original Kolmogorov's hypotheses, which is responsible for the departure from K41 predictions for structure functions scalings. To account for these anomalous exponents, original Kolmogorov's self-similar hypotheses had to be refined. Kolmogorov himself proposed such a refinement in 1962 (Kolmogorov, 1962) incorporating Oukhov's suggestion of strongly nonGaussian fluctuations of the energy dissipation rate (Obukhov, 1962) after Landau objected the averaged energy dissipation might not be a sufficient parameter to describe turbulent fields as assumed in original K41 hypotheses. Since then, several other descriptions of intermittency have emerged, among which the most popular has become the multi-fractal description introduced by Parisi and Frisch in 1985 (Parisi and Frisch, 1985).

\subsection{Lagrangian K41 phenomenology}

K41 phenomenology, originally developed in the Eulerian framework, can be extended to the Lagrangian framework, by introducing the notion of temporal velocity increments along particles trajectories (which is the Lagrangian counter-part of the Eulerian spatial increments of the velocity field): $\delta_{L} \boldsymbol{v}(\tau)=\boldsymbol{v}(t+\tau)-\boldsymbol{v}(t)$. Here, $v$ refers to the Lagrangian velocity, measured for each fluid particles along its trajectory. Statistics will be assumed to be stationnary, hence independent of time $t$, and only dependent on the time increment $\tau$. Spanning statistical behaviors at different values of $\tau$ allows to investigate the multi-scale temporal dynamics of Lagrangian temporal. This multiscale characterization is generally achieved by considering the dependency of the statistical moments of Lagrangian increments with $\tau$. These define the Lagrangian function structures: $S_{p}^{L}(\tau)=<\delta_{L} \boldsymbol{v}(\tau)^{p}>$. As for the Eulerian approach, the second order moment $(p=2)$ is of particular interest as it is directly related to the Lagrangian correlation function $R_{v v}^{L}(\tau)=\sigma_{v}^{2}(1-$ $\left.S_{2}^{L}(\tau) / 2 \sigma_{v}^{2}\right)$, which is itself related to the Lagrangian velocity spectrum by a simple temporal Fourier transform. The same dimensional analysis arguments as in K41 phenomenolgy allow to 
determine the scalings for the Lagrangian structure function at temporal inertial scales, under the self-similar hypothesis, where $\epsilon$ is the only relevant physical parameter:

$$
S_{p}^{L}(\tau) \propto(\epsilon \tau)^{p / 2} \text { for } \tau_{\eta} \ll \tau \ll T_{L}
$$

This predicts in particular that at inertial scales the second order Lagrangian structure function is linear in $\tau: S_{2}^{L}(\tau) \propto \epsilon \tau$ (which the Lagrangian counter-part of the Eulerian relation $S_{2}^{E}(r) \propto(\epsilon r)^{2 / 3}$ ) and equivalently the Lagrangian velocity spectrum $S_{2}^{L}(\omega)=C_{0} \epsilon^{1 / 2} \omega^{-2}$ (which the Lagrangian counter-part of the Eulerian spectrum $\left.S p^{E}(k) \propto \epsilon^{2 / 3} k^{-5 / 3}\right)$, with $C_{0}$ some universal constant.

As for the Eulerian case, the simple K41 scaling for the Lagrangian strcuture functions 1 is known to fail for large moments, due to intermittency effects. This results in anomalous exponents such that for $S_{p}^{L}(\tau)=(\epsilon \tau)^{\zeta_{p}}$, with $\zeta_{p}<p / 2$ (Mordant et al. (2001); Xu et al. (2006)).

\subsection{Lagrangian dispersion}

One of the most classical problems of Lagrangian turbulence concerns the question of particles dispersion, with two main important aspects: (i) diffusion of single particles from a point sourve (also known as Taylor problem) and (ii) the relative dispersion of pairs of particles (also known as the Richardson problem). The case of more than one or two particles has also been more recently addressed by considering triads and tetrads of particles (Chertkov et al. (1999)). We will briefly recall here the main results of the Taylor problem, by considering the dependency of the particle mean square displacement from a point source. The case of relative dispersion will be further detailed below in section 4 .

Consider a point source where particles are continuously released, at which rate particles move away from the source? Taylor addressed this question in 1922 in a seminal article ((Taylor, 1922)) where he proposed a solution of this problem based on the asumption that the Lagrangian correlation function is an exponential function: $R_{v v}^{L}(\tau)=\sigma_{v}^{2} e^{-\tau / T_{L}}$, where $T_{L}$ is the Lagrangian correlation time of the velocity, also known as the Lagrangian integral time. It can be noted that at short times $\left(\tau \ll T_{L}\right)$ such an exponential behavior is consistent with K41 scaling as $R_{v v}^{L}\left(\tau \ll T_{L}\right)=\sigma_{v}^{2}\left(1-\tau / T_{L}\right)$ implies $S_{2}\left(\tau \ll T_{L}\right) \propto \tau$ (ought to the relation $\left.R_{v v}^{L}(\tau)=\sigma_{v}^{2}\left(1-S_{2}^{L}(\tau) / 2 \sigma_{v}^{2}\right)\right)$. However, such a simple exponential correlation function cannot account for small scale dissipative scales $\left(\tau \ll \tau_{\eta}\right)$, where the trivial, purely kinematic scaling $S_{2}^{L}(\tau)=\sigma_{a}^{2} \tau^{2}$ (with $\sigma_{a}^{2}$ is the acceleration variance) is expected as at vanishing time increment $\tau \rightarrow 0$ velocity increments are simply representative of acceleration. This is due to the fact that a simple exponential decorrelation conveys only one characteristic timescale (here the Lagrangian integral timescale $T_{L}$ ). Extensions to this simple approximation have been formulated since then, in the context of autoregressive stochastic models, as the two-time stochastic model by Sawford (1991), which accounts for small scales effects by introducing a double exponential correlation function. For the sake of simplicity, we will briefly present here the result by Taylor without this additional small scale corrective term (hence assuming simply an exponential correlation function) and considering only one component (let say $x$ ) of the displacement. Let $x_{0}$ be the initial source point. The mean square displacement can then be written as $\left\langle\left(x(t)-x_{0}\right)^{2}\right\rangle=\int_{0}^{t} \int_{0}^{t} R_{v v}\left(\tau^{\prime}-\tau\right) \mathrm{d} \tau \mathrm{d} \tau^{\prime}$. Assuming an exponential Lagrangian correlation function for the velocity, leads to $\left\langle\left(x(t)-x_{0}\right)^{2}\right\rangle=2 \sigma_{v}^{2} T_{L}\left(t+T_{L} e^{-t / T_{L}}-T_{L}\right)$, with two importnat asymptotic behaviors:

$$
\begin{gathered}
\left\langle\left(x(t)-x_{0}\right)^{2}\right\rangle=\sigma_{v}^{2} t^{2} \quad \text { for } t \ll T_{L} \\
\left\langle\left(x(t)-x_{0}\right)^{2}\right\rangle=2 \sigma_{v}^{2} T_{L} t \text { for } t \gg T_{L} .
\end{gathered}
$$

This simple result can be of interest for instance to predict the growth rate of a puff of pollutants released from a point source (for instance an industrial chimney). It shows that the mean growth rate of the puff (which can be estimated as $\left\langle\left(x(t)-x_{0}\right)^{2}\right\rangle^{1 / 2}$ ) is directlty related to the Lagrangian correlation function (or equivalently to the Lagrangian second order structure function or Lagrangian velocity spectrum). The two asymptotic behaviors discussed above reveal that the 
puff will first grow ballistically (its size increasing linearly with time for timescales smaller than the Lagrangian correlation time) before transienting towards a brownian like behaviour where its size increases as the $\sqrt{t}$ for larger timescales. The evolution of the fluctuations of pollutants distribution within the cloud is however related to multi-particles statistics (Falkovich et al., 2001); in particular the evolution of the variance of the pollutants distribution is directly related to the relative dispersion problem, which will be discussed later in section 4 .

After this brief review of some of the main properties of Lagrangian turbulence, we discuss in the next sections two more specific questions related to Lagrangian properties of turbulence of interest for more complex flows than homogeneous isotropic turbulence, as geophysical flows, where anisotropy and two-dimensionalisation effects can affect the way particles are transported and dispersed by the turbulence.

\section{On the validity of Kolmogorov's local isotropy hypothesis}

\subsection{The local isotropy hypothesis}

Kolmogorov's original self-similar hypotheses assumed that well away from any boundaries or singularities, the statistics of turbulent flows should be universal at length and timescales that are small compared with the injection of energy into the flow and that averaged energy dissipation $\langle\epsilon\rangle$ should be the only relevant parameter for inertial range dynamics. As briefly discussed in the previous section, intermittency and anomalous scaling corrections reveal the limitation of considering solely the averaged dissipation $\langle\epsilon\rangle$ in particular when high order moment statistics of velocity increments are considered. Evidence of intermittency led Kolmogorov himself to reconsider, in 1962, the original formulation of self-similar hypotheses in order to account for local fluctuations of the dissipation rate $\epsilon$. Another fundamental aspect of Kolmogorov's hypotheses concern the local-isotropy approximation which state that if the small-scale statistics are to be universal, they must be independent of the large-scale flow structure. In particular, K41 assumes that at inertial and dissipative scales the turbulence should "forget" any preferred direction of the large-scale flow and that the small-scale fluctuations should be statistically homogeneous and isotropic; this is the local isotropy hypothesis. Models and simulations of turbulence therefore commonly assume such isotropic conditions. Real flows, however, are never homogeneous and isotropic at large scales. Careful study of the effects of large-scale anisotropy on the small-scale turbulent fluctuations is therefore very important for understanding the behavior of turbulent flows in real systems.

Persistent anisotropy at small scales has been noted previously in Eulerian studies of homogeneous shear flows (Pumir and Shraiman, 1995; Garg and Warhaft, 1998) and in the context of the SO(3) symmetry group (Biferale and Procaccia, 2005). Available data is however usually limited to low or moderate Reynolds number and the trend of the persistence of small-scale anisotropy with Reynolds number remains unclear. Is small-scale isotropy somehow recovered for sufficiently large Reynolds number? This remains an open question which has been recently tackled from the Lagrangian point of view, by addressing the question of the relevance of the K41 hypothesis of local isotropy in a von Kármán experiment (Ouellette et al., 2006), which exhibits a strong imposed large scale anisotropy and exploring the recovery of isotropy across scales using high resolution measurements of the second order Lagrangian velocity structure function and spectrum. Von Kármán geometry is indeed particularly well suited for this study as it has a pronounced large-scale anisotropy (the geometry of the system imposes a large scale axisymetric forcing) at the same time as it produces easily very large Reynolds number turbulence.

The effects of this large-scale anisotropy are investigated based on the comparison of different projections of the second order Lagrangian structure function $D_{2, i j}^{L}(\tau)=<\delta u_{i}(t+\tau) \delta u_{j}(t)>$. Axisymmetric turbulence has been the subject of prior theoretical works (Batchelor, 1946; Chandrasekhar, 1950; Ould-Rouiss, 2001), but has not yielded any experimentally verifiable prediction similar to those made by K41. Considering the second order structure function is of particular interest since it is known not to be significantly affected by intermittent corrections compared to higher order structure functions. This allows to unambiguously investigate effects of anisotropy limiting other spurious effects. K41 phenomenology predicts that the Lagrangian structure func- 


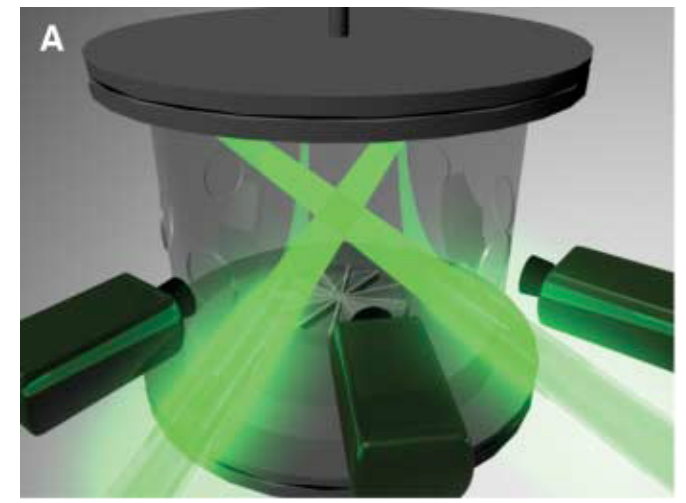

Figure 2. Sketch of the experimental setup used by Ouellette et al. (2006). Three high-speed cameras were used to record the three-dimensional tracks of tracer particles in intense turbulence. The particles were illuminated by two high-power lasers.

tion tensor should scale as $D_{2, i j}^{L}(\tau)=C_{0} \epsilon \tau \delta_{i j}$ in the inertial range (with $i$ and $j$ representing the three spatial coordinates). According to K41 universality hypotheses, the structure function should be isotropic and $C_{0}$ should have a universal value for all turbulent flows (at least in the limit of high Reynolds number). It is an important parameter in stochastic models of turbulent transport and dispersion (RODEAN, 1991; Sawford, 1991; Weinman and Klimenko, 2000) and is, remarkably, also connected to the structure functions of the fluctuations of a scalar field passively advected by the turbulence (Sawford, 2001). Previously measured values of $C_{0}$ range from 2.1 to 7.0, in part because Lagrangian experiments, where the trajectories of individual fluid particles are followed, have historically been very difficult. Hanna HANNA (1981) measured the Lagrangian spectra in the atmospheric boundary layer using neutrally buoyant balloons, but acknowledged significant (as much as 50\%) uncertainty in the measurements, reporting a value of $4 \pm 2$ for $C_{0}$. Lien et al. (1998) measured 1D spectra using large floaters (roughly $1 \mathrm{~m}$ in scale) in the oceanic boundary layer. Due to the considerable noise in their measurements, they were only able to estimate that the value of $C_{0}$ lies somewhere between 3.1 and 6.2. Mordant et al. (2001) measured the radial Lagrangian structure function in a laboratory acoustic particle tracking experiment in a counter-rotating disk device similar to ours and obtained a maximum value of 4 for $C_{0}$, which may be depressed due to the filtering effect of their relatively large tracer particles. Lien and D'Asaro (2002) have estimated a value of 5.5 from the spectral data published by Mordant et al. (2001).

\subsection{The local isotropy hypothesis in large scale anisotropic flows}

To address the question of the anisotropy of $C_{0}$, figure $3 \mathrm{a}$, shows the full structure function tensor measured at $R_{\lambda}=815$ by Ouellette et al. (2006). Two features of this tensor are particularly noteworthy. We see very short plateau regions for all three diagonal components of the structure function tensor, consistent with the K41 scaling, though without a fully developed Lagrangian inertial range. It is clear, however, that this tensor is not isotropic, contradicting the K41 hypothesis of local isotropy. The $z z$ component, measured in the axial direction of the cylindrical flow chamber, shows a peak value roughly $25 \%$ lower than that of the $x x$ and $y y$ components, measured in the radial direction. The $x x$ and $y y$ components are identical within experimental precision, reflecting the axisymmetry of the large-scale flow. We note that the peak values of the compensated structure functions occur at very short times, less than a factor of 10 larger than the Kolmogorov time $\tau_{\eta}$, the characteristic timescale of the fastest turbulent motion. It is also interesting to note that, though it is reduced, anisotropy remains even at the smallest scales.

The anisotropy can also be investigated via the Lagrangian velocity spectrum. Though the spectrum is essentially nothing but the Fourier space representation of the second order structure function just discussed it can emphasize different information of the analyzed data (Davidson and Pearson, 2005), in particular regarding the scale by scale anisotropy. Following K41 phenomenology, the Lagrangian spectrum should scale as $E_{L}(\omega)=B_{0} \epsilon \omega^{-2} \delta_{i j}$ within the range of inertial time scales, and like the structure function it should be expected to become isotropic at small (inertial 
(a)

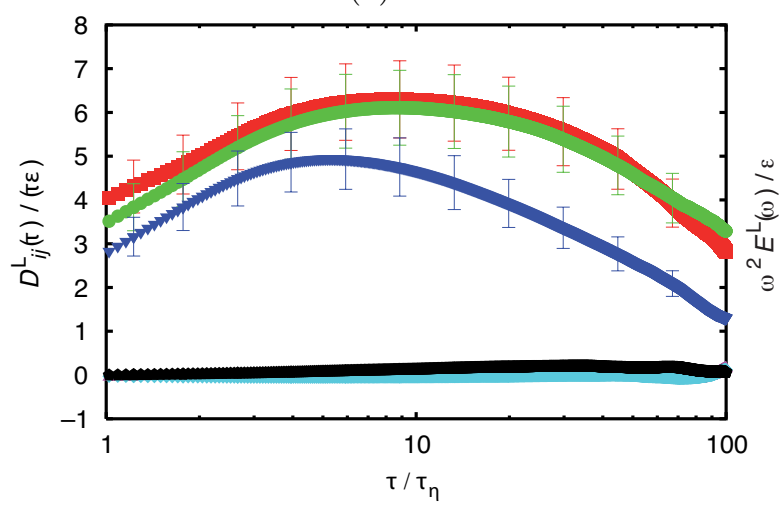

(b)

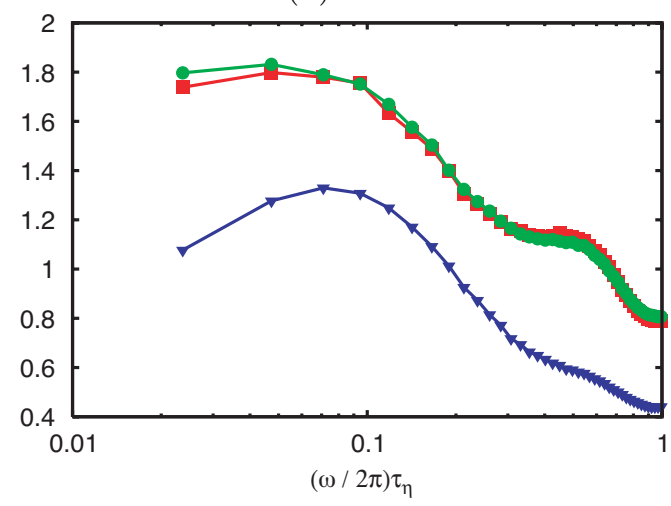

Figure 3. (a) The $x x(\mathbf{\bullet}), y y(\bullet)$ and $z z(\mathbf{v})$ components of the compensated Lagrangian structure function at $R_{\lambda}=815$. The other symbols show the off-diagonal components. The time axis has been normalized by the Kolmogorov time. The relative magnitude of the radial and axial components reflects the anisotropy of our large-scale flow. (b) Compensated Lagrangian velocity spectra at $R_{\lambda}=690$ in the $x$-direction $(\mathbf{-})$, y-direction $(\bullet)$ and z-direction $(\mathbf{v})$. By scaling the spectra by $\epsilon \omega^{-2}$, we expect to see a plateau in the inertial range with value $B_{0}$. The frequency axis has been scaled by the Kolmogorov frequency. As before, we note that the difference in magnitude between the radial spectra and the axial spectrum reflects the large-scale structure of our flow. The bump in the spectrum at high frequencies is due to noise in the measurements, but the inertial range behaviour is unaffected.

and dissipative) scales. The constant $B_{0}$, which is related to $C_{0}$ simply by a factor of $\pi\left(C_{0}=\pi B_{0}\right)$, is the Lagrangian analog of the Kolmogorov constant. The spectral representation also exhibits the same pronounced anisotropy between, the axial and the radial components.

\subsection{The influence of increasing Reynolds number}

The anisotropy found between the radial and axial components of both the structure function and the spectrum persists at all the investigated Reynolds numbers. Figure 4a, shows values of $C_{0}$ determined from the plateaux of the compensated structure functions as a function of the Reynolds number $R_{\lambda}$. For both the axial and radial structure functions, we also observe that $C_{0}$ increases weakly with Reynolds number. It is encouraging to note that figure 4 shows that the $C_{0}$ estimates seem to saturate as the Reynolds number increases; this result suggests that we may measure true inertial range behavior at high Reynolds number despite the very short scaling range of the structure function. To model this Reynolds number dependence of $C_{0}$ in homogeneous isotropic turbulence, Sawford (1991) has empirically fitted the trends obtained from direct numerical simulations and proposed that

$$
C_{0}=\frac{C_{0}^{\infty}}{1+A R_{\lambda}^{-1.64}},
$$

where $C_{0}^{\infty}$ is the asymptotic value of $C_{0}$ at infinite Reynolds number. From simulation data, Sawford estimated that $C_{0}^{\infty} \simeq 7$ and $A \simeq 365$. Fits of this function to our $C_{0}$ data are shown in figure 4a, where Sawford's fit has being applied to the different components. We find that $C_{0}^{\infty}=6.2 \pm 0.3$ for the radial structure functions and $C_{0}^{\infty}=5.0 \pm 0.4$ for the axial structure function (similar values for $A$ are found in both cases).

The measurements of $C_{0}$ remain anisotropic even at the highest Reynolds number investigated. Figure $4 \mathrm{~b}$, shows the ratio of the radial measurements to the axial measurements. The anisotropy drops weakly with Reynolds number, but the decrease is very slow and the anisotropy remains strong even at the highest Reynolds number investigated. Taken together, these results suggest that any symmetries (or lack thereof) present at the large scales of the flow will also be reflected in the small-scale turbulent fluctuations. Clearly, therefore, great care must be exercised when applying the results of isotropic turbulence theory to real experimental, industrial and natural flows. For 
(a)

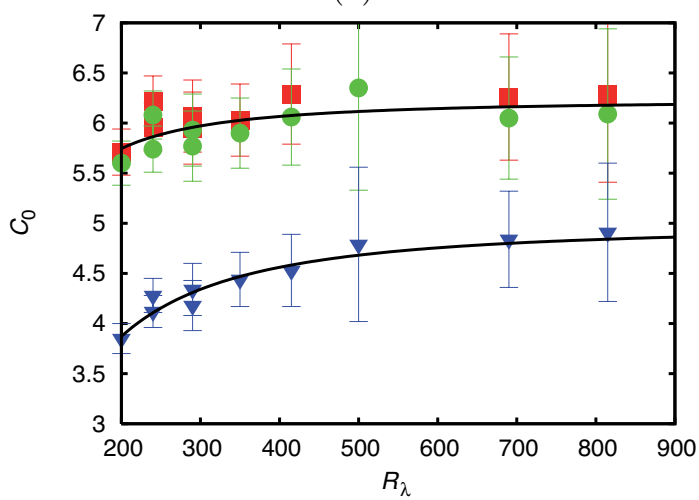

(b)

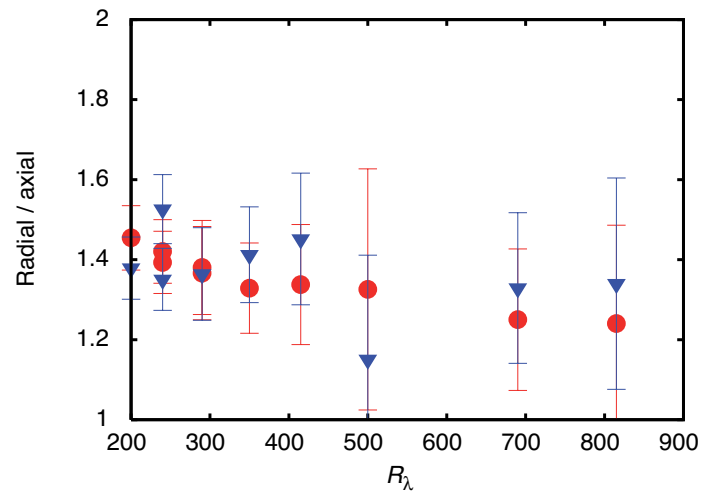

Figure 4. (a) Measurements of $C_{0}$ from the Lagrangian structure function tensor for the xx component ( $\mathbf{\square})$, yy component $(\bullet)$ and zz component $(\mathbf{v})$ as a function of Reynolds number. The zz component $C_{0}$ values are smaller than those measured for the two radial components, presumably due to the large-scale axisymmetry of our flow. $C_{0}$ is observed to increase weakly with Reynolds number. The solid lines are fits of Sawfords model 4 for the Reynolds number dependence of $C_{0}$ Sawford (1991). We note that due to the time resolution in the $R_{\lambda}=500$ data run, we encountered large uncertainties and were not able to measure a $C_{0}$ value from the xx component. We have therefore not included the $R_{\lambda}=500$ data points in the fits of 4 . (b) The ratio of the radial to the axial measurements of $C_{0}$ as a function of Reynolds number from both the structure function $(\bullet)$ and the spectrum $(\mathbf{v})$. While the anisotropy decreases weakly with increasing Reynolds number, the measurements remain far from isotropic even at the highest Reynolds numbers measured.

instance, any climate or pollutant transport models must take the significant anisotropies present in the atmosphere into account. The significant difference between the scaling constants measured in the radial and axial directions reflects the large-scale axisymmetry in the flow. Extrapolation based on the Sawford's model even predicts a persistence of small-scale anisotropy in the limit of infinite $R_{\lambda}$. Though such an extrapolation is highly speculative as long as the limits of validity of Sawford's fit are not well controlled, it is clear that the recovery of isotropy (if any) with increasing Reynolds number is much slower than expected. We do, however, observe K41 scaling ranges for both the Lagrangian structure function and spectrum, suggesting that while our results contradict the K41 hypothesis of local isotropy, the K41 scaling hypotheses are fulfilled for second order statistics.

It is also intriguing to notice that in spite of the clear persistence of small scale anisotropy seen on the second moment of Lagrangian velocity increments, this anisotropy does not seem to significantly affect the second order moment of the Lagrangian position increments (i.e. the root mean separation investigated in the relative dispersion problem), for which the Btachelor superdiffusive law (which is established using a local isotropy hypothesis), fits almost perfectly the data. As the root mean separation between particles is directly related to the second moment of Eulerian velocity increments, this observation suggests that Lagrangian statistics may be more sensitive to small scale anisotropy than Eulerian's.

To summarize, this investigation shows that the local isotropy hypothesis, generally considered in models and simulations might be more controversial than usually believed. Large scale anisotropy appears to persist at small scales, even in the limit of large Reynolds numbers (approaching $R_{\lambda} \sim 10^{3}$ ).

\section{Turbulent dispersion of tracer particles}

\subsection{The turbulent pair dispersion problem}

Molecules in a quiescent fluid spread due to molecular diffusion. If we consider a small spherical patch of tagged molecules, this results in an isotropic and homogeneous growth of the patch. 
At a microscopic level this expansion is due to random uncorrelated collisions induced by the thermal agitation of the molecules. At a macroscopic level this mechanism results in a Fickian diffusion process where the local concentration $C$ of tagged molecules diffuses according to the simple equation $\partial C / \partial t=K \Delta C$, where $K$ is the molecular diffusivity, with units $\left[\mathrm{m}^{2} \cdot \mathrm{s}^{-1}\right]$. In elementary kinetic gas theory, the connection between microscopic and macroscopic descriptions is for instance given by the relation $K \propto l v_{T}$ (with $l$ a characteristic correlation length of particles trajectories, typically given by the mean free path and $v_{T}$ the thermal agitation velocity of the molecules). A fundamental property of such a Fickian process concerns the linear growth with time $t$ of the mean square separation $\left\langle\vec{D}^{2}>\propto K t\right.$ between any two molecules in the patch, what is generally referred to as normal diffusion. Normal molecular diffusion alone is very inefficient to mix and disperse usual species: for instance, molecular diffusivity of carbon dioxyde in air is $16 \cdot 10^{-6} \mathrm{~m}^{2} \cdot \mathrm{s}^{-1}$, meaning that molecules separate at a rate of only a few millimeters per second.

In macroscopic flows, the slow effect of molecurlar diffusion is compensated by the efficiency of turbulence to mix and disperse substances and particles. At the largest scales, the uncorrelated turbulent structures act in a similar way (i.e. normally diffusive) as molecular diffusion, but with an enhanced diffusion coefficient $K_{\text {turb }} \propto L \sigma$ with $L$ the turbulence correlation length scale and $\sigma$ the turbulent fluctuating velocity (standard deviation of the turbulent velocity field). In atmospheric dispersion for instance, the turbulent correlation length can be as large as of the order of hundreds of meters (let us take $100 \mathrm{~m}$ as an order of magnitude) with velocity fluctuations typically of the order of meters per second in normal conditions (let us take $1 \mathrm{~m} / \mathrm{s}$ as an order of magnitude), leading to a turbulent diffusivity coefficient $K_{\text {turb }}$ of the order of $30 \mathrm{~m}^{2} \cdot \mathrm{s}^{-1}$, meaning that fluid particles separate at a rate of several meters per second, hence many orders of magnitude larger than molecular diffusion. The efficiency of turbulent diffusion therefore relies on the capacity of a substance to spread thanks to the uncorrelated motion of large scale turbulent eddies. However, if we consider the dispersion of a patch initially much smaller than the turbulent correlation scale $L$ (for instance a patch with an initial dimension within the inertial range of the carrier turbulence, hence much smaller than the energy injection scale $L$, and larger than the dissipation scale $\eta$ ), another mechanism is necessary to allow the patch to grow first at sufficiently large scales to eventually undergo the effect of uncorrelated turbulent diffusion. Such an inertial scale mechanism is ensured by the super-diffusive nature of turbulence at inertial scales. Processes where the mean square separation grows faster than in normal diffusion (i.e. $<\vec{D}^{2}>\propto t^{\alpha}$, with $\alpha>1$ ) are called super-diffusive. Unlike normal diffusion, super-diffusion is generally associated with an heterogeneous and a non-gaussian growth of the spreading patch.

\subsection{Batchelor and Richardson regimes for pair dispersion}

Predictions for the superdiffusivity of pair dispersion in turbulence date back to 1926, when Richardson (1926) suggested that the mean square separation between two particles in the range of inertial scales should grow superdiffusively in time as $t^{3}$. By applying Kolmogorov's scaling phenomenology, Oboukhov (1941) specified that in the inertial range of turbulence, where the only relevant flow parameter is the energy dissipation rate per unit mass $\epsilon$, the mean square pair separation should grow as $\left\langle\Delta^{2}(t)\right\rangle=g \epsilon t^{3}$, whith $g$ a universal constant. An important feature of the Richardson-Obukhov superdiffusive prediction is that the separation rate is cubic in time and independent of initial separation. The absence of initial separation is however more an assumption than a consequence of Richardson-Obukhov's prediction, since the $t^{3}$ law can indeed be derived by simple dimensional analysis only if initial separation is considered as an irrelevant parameters. In this case the only relevant parameters being $\left\langle\Delta^{2}(t)\right\rangle, \epsilon$ and $t$, Richardson-Obukhov is a direct result of Pi-Buckingam theorem as the only possible relation dimensionally consistent.

In 1950 Batchelor (1950) refined Richardson and Obukbhov's work by considering initial separation as a possible relevant parameter of the problem. In this case, he predicted that two different dispersion regimes should exist for the dispersion of particles with initial separation at inertial range scales : for times shorter than a characteristic timescale $t_{0}$, which depends on the initial separation of the pair, the mean square separation should grow ballistically as $t^{2}$; the $t^{3}$ law being expected only for times longer than $t_{0}$. More precisely, if $\Delta(t)$ is the separation of two fluid elements at time $t$ and defining $\Delta_{0}$ as the initial separation between the fluid elements, Batchelor predicted that for $\Delta_{0}$ in the inertial range 


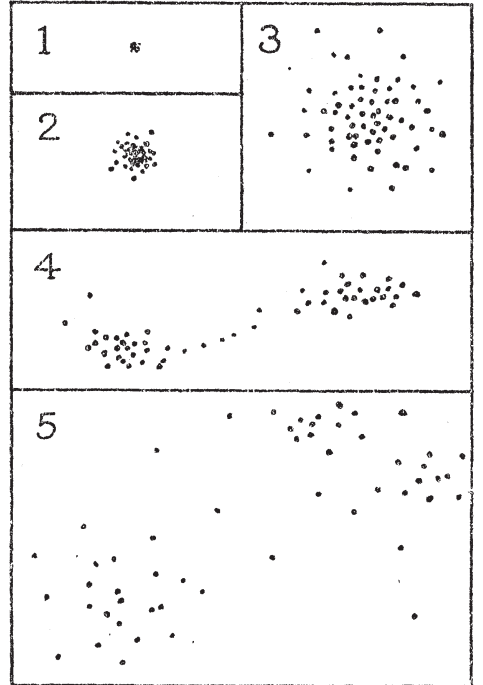

(a)

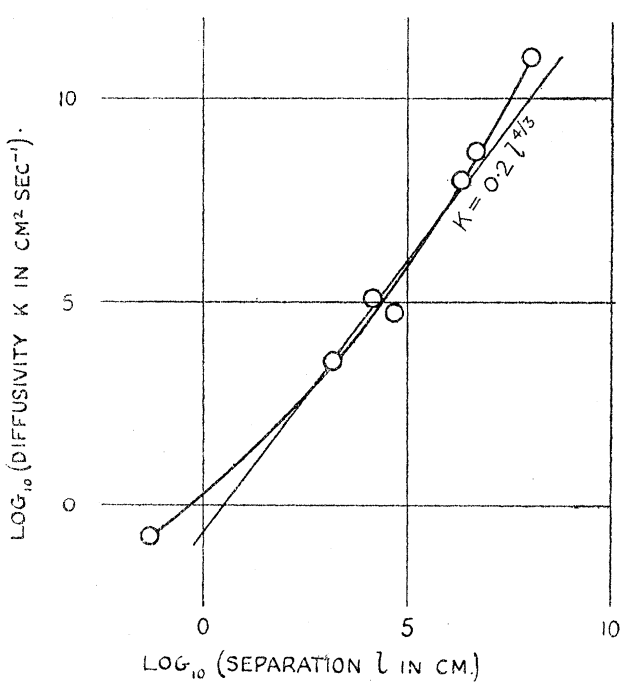

(b)

Figure 5. (a) Qualitative illustration of the non-normal dispersion of a dense cluster of particles as proposed in Richardson's original 1926 article. (b) Original empirical derivation of the "4/3rd" law by Richardson. (Both figures are taken from Richardson's seminal article on relative dispersion (Richardson, 1926)).

$$
\left\langle\left[\vec{\Delta}(t)-\vec{\Delta}_{0}\right]^{2}\right\rangle= \begin{cases}\frac{11}{3} C_{2}\left(\epsilon \Delta_{0}\right)^{2 / 3} t^{2} & \text { if } t \ll t_{0}=\left(\frac{\Delta_{0}^{2}}{\epsilon}\right)^{1 / 3} \\ g \epsilon t^{3} & \text { if } t_{0} \ll t \ll T_{L} \\ D t & \text { if } T_{L} \ll t\end{cases}
$$

where $C_{2}$ is the universal constant in the inertial range scaling law for the Eulerian second-order velocity structure function, which has a well-known value of approximately 2.1 (Sreenivasan, 1995) (interestingly, this relation directly bridges Eulerian and Lagrangian approaches). Note that the first equation in 5 arises from a purely kinematic argument, trivially stating that at short times, a Taylor expansion of particles separation time dependency gives

$$
\left\langle\left[\vec{\Delta}(t)-\vec{\Delta}_{0}\right]^{2}\right\rangle=S_{2}\left(\Delta_{0}\right) t^{2} \quad \text { if } \quad t \ll t_{0},
$$

with $S_{2}\left(\Delta_{0}\right)=\left\langle\left(\delta_{\Delta_{0}} \vec{u}\right)^{2}\right\rangle$ the second order Eulerian structure function estimated at a scale given by the initial separation $\Delta_{0}$. In the classical cascade model of turbulence, $t_{0}$ corresponds to the eddy turnover time at the scale $\Delta_{0}$ and may be interpreted as the time for which the two fluid elements "remember" their initial relative velocity as they move in the same eddy of size $\Delta_{0}$. For times on the order of $t_{0}$, this eddy breaks up, and the growth of the pair separation is then expected to undergo a transition to Richardson-Obukhov scaling. According to this prediction, at short times (namely $t \ll t_{0}$ ), relative dispersion is ballistic and initial separation dependent, while it accelerates for $t_{0} \ll t \ll T_{L}$ at the same time as it looses the memory of its initial separation. For times much larger (namely $t \gg T_{L}$ ) a Brownian-like dispersion is recovered as the two particles evolve then without any correlation. The aim of this study was to investigate this scenario by a systematic analysis of dispersion of pairs of particles in a highly turbulent flow, emphasizing the role of initial separation of the particles.

In his seminal 1926 article, Richardson gave an interpretation of turbulent $t^{3}$ super-diffusion in terms of a non-Fickian process which could be locally modeled as a normal diffusion process, but with a scale dependent diffusion coefficient which depends on particles separation $D$, according to the celebrated Richardson's 4/3rd law: $K(D) \propto D^{4 / 3}$. Richardson conjectured such a scale 


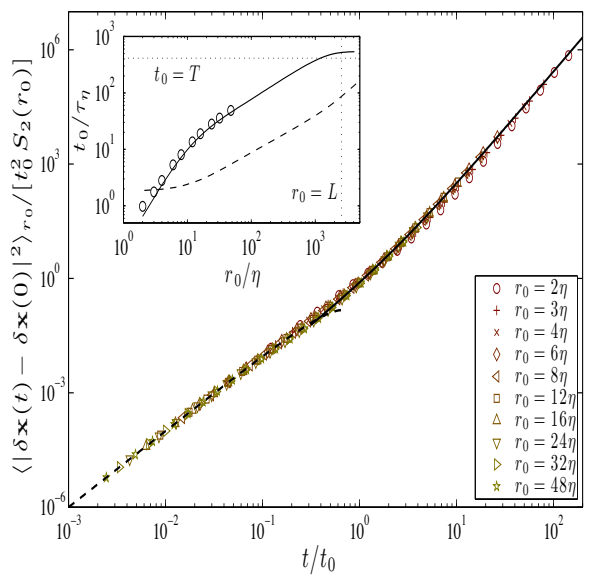

(a)

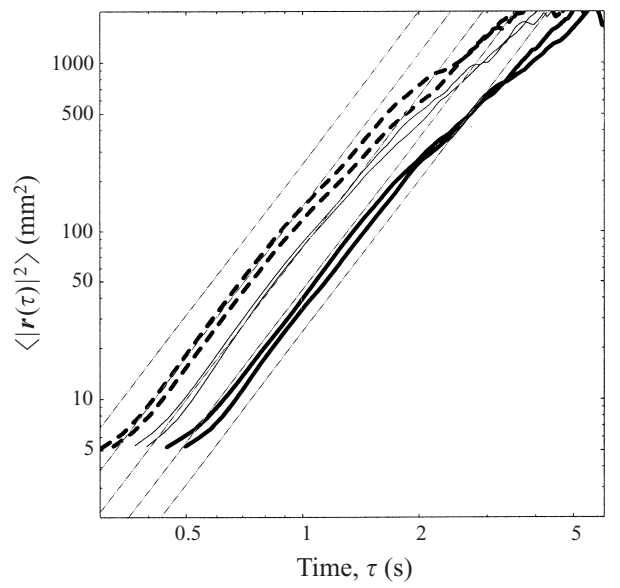

(b)

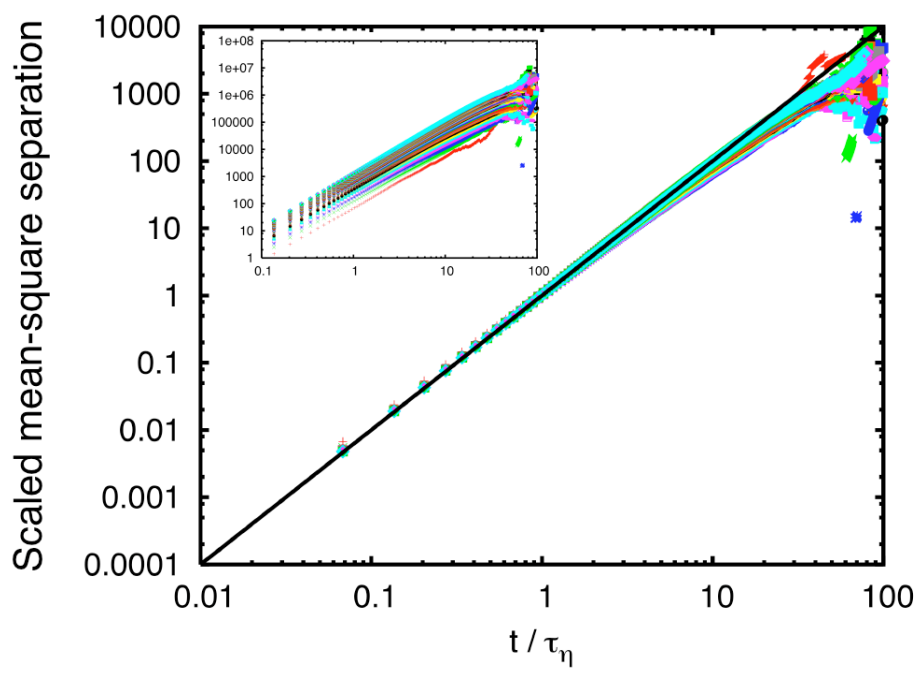

(c)

Figure 6. Growth of the mean square separation of particle pairs in: (a) direct numericam simulations by Bitane et al. (Bitane et al., 2012) (time is non-dimensionalized by $t_{0}=S_{2}\left(D_{0}\right) / 2 \epsilon$ which within K41 framework is porportional to $\epsilon^{-1 / 3} D_{0}^{2 / 3}$, different symbols correspond to different initial separations) ; at short time the separation follows a ballistic regime a la Batchelor, while at long times the separation is cubic, a la Richardson, with a transition occuring for times $t \simeq t_{0}$. (b) Experiments by Ott \& Mann (Ott and Mann, 2000) ; time in this plot has been shifted by virtual time origin $T_{0}$ to emphasize the cubic behavior. (c) experiments by Bourgoin et al. (Bourgoin et al., 2006) where the ballistic regime is robustly observed, without any adjustable parameter.

dependent diffusive scenario from an empirical short time, scale by scale, analysis of local diffusion properties over a wide range of phenomena, from diffusion of oxygen intro nitrogen, to the diffusion of cyclones in the atmosphere (Richardson, 1926) (figure 5b), such that at each scale the mean square separation could be locally written as $D^{2} \propto K(D) t$. It is now accepted that his derivation of the $4 / 3$ rd law was at the same time fortuitous and the result of his unique intuition (Sawford, 2001). Richardson also showed that such a non-Fickian diffusion resulted in a cubic super-diffusive growth of the mean square separation of pairs of particles according to the law $\left\langle D^{2}\right\rangle=g \epsilon t^{3}$, where $\epsilon$ is the turbulent energy dissipation rate and $g$ a universal constant since known as the Richardson constant. 
The Richardson constant $g$ in eq. 5 is one of the most fundamental constants in turbulence (together with the Kolmogorov constant $C_{K}$ ). It plays a major role in turbulent dispersion and mixing processes. However, in spite of its importance, it is only recently that estimations of its value started to converge towards a well accepted value [(Sawford, 2001; Salazar and Collins, 2009)]. This is due to the difficulty to observe experimentally Richardson's superdiffusion [(Sawford, 2001; Bourgoin et al., 2006)]. Until recently, best estimates for $g$ still spanned several orders of magnitude. Most recent high resolution direct numerical simulations seem to point toward an estimate of $g \sim 0.5-0.6$ (Bitane et al., 2012; Boffetta and Sokolov, 2002a) (fig. 6a). This value is also consistent with experiments by Ott \& Mann (Ott and Mann, 2000) in homogeneous and isotropic turbulence at a moderate Reynolds number $R_{\lambda} \simeq 100$, based on Taylor micro-scale (fig. $6 \mathrm{~b}$ ).

Apart from this experiment, high resolution data in well controlled laboratory experiments is still very scarce. This is very likely due to the difficulty in accessing sufficiently long tracks in Lagrangian measurements, allowing to unambiguously observer the long-term cubic regime $a$ la Richardson. Recent experiments by Bourgoin et al. (Bourgoin et al., 2006) report highly resolved particle tracking measurements of relative dispersion at high Reynolds numbers (up to $R_{\lambda} \simeq 800$ ), although the track lengths in this experiment where too short to actually observe the $t^{3}$ Richardson regime and only the early ballistic regime $6 \mathrm{c}$ was observed.

In the next subsection, a simple phenomenology for pair dispersion is proposed which elucidates many aspects of the problem, in particular regarding the origin of the cubic super-diffusive longterm regime and its deep connection with fundamental properties of the turbulent energy cascade. We also discuss the expected consequences for the case of $2 \mathrm{D}$ turbulence, relevant for large-scale geophysical flows.

\subsection{A simple ballistic phenomenology of turbulent superdiffusion}

A recent work (Bourgoin, 2015) suggests that a scale dependent ballistic phenomenolgy, rather than a scale dependent diffusive phenomenology as originally proposed by Richardson, may be best suited to quantitatively describe turbulent super-diffusion. The idea is quite simple. Let consider an ensemble of particles with an initial mean square separation $D_{0}^{2}$. In the short term, the particles will separate ballistically according to the kinematic relation 6 . Let $t_{0}$ be the typical duration of the ballistic initial ballistic growth. Experimental and numerical evidence discussed in the previous section confirme Batchelor's suggestion that $t_{0} \propto \epsilon^{-1 / 3} D_{0}^{2 / 3}$. Following Bitane et al. we can equivalently write $t_{0}^{\prime}=\alpha S_{2}\left(D_{0}\right) / 2 \epsilon$, with $\alpha$ a non-dimensional parameter we shall call persistence parameter as it quantifies the persistence of the ballistic regime. The main idea behind the ballistic cascade mechanism, is illustrated in figure 7. It is based on the trivial idea that if an ensemble of particles with initial mean square separation $\vec{D}_{0}^{2}$ starts to disperse ballistically, with a separation rate $S_{2}\left(\vec{D}_{0}\right)$ over a given period $t_{0}^{\prime}$ after which it reaches a new mean square separation ${\overrightarrow{D_{1}}}^{2}$, instead of considering a sudden transition towards an enhanced cubic dispersion regime $\grave{a}$ la Richardson (as in eq ??), the ballistic process can instead be iterated starting from the new mean square separation $\vec{D}_{1}^{2}$, with a new separation rate $S_{2}\left(\vec{D}_{1}\right)$ over a period $t_{1}^{\prime}$ and so on. Thus, in this scenario the time evolution of particles mean square separation is simply described by the iterative process :

$$
D_{k+1}^{2}=D_{k}^{2}+S_{2}\left(D_{k}\right) t_{k}^{2}\left(D_{k}\right)
$$

where $D_{k}^{2}=<\left|\vec{D}_{k}\right|^{2}>$ represents the mean square separation of pairs after the $k^{t h}$ iteration step, $t_{k}^{\prime}\left(D_{k}\right)$ is a scale dependent "time of flight" characteristic of the duration of the ballistic motion at step $k+1$. For the case of turbulent flows, $S_{2}\left(D_{k}\right)$ and $t_{k}^{\prime}\left(D_{k}\right)$ will be prescribed later by imposing K41 scalings.

A concrete implementation of the iterative scheme (7) requires the expressions for the scale dependent separation rate $S_{2}\left(D_{k}\right)$ and the ballistic time of flight $t_{k}^{\prime}\left(D_{k}\right)$ to be specified. For particles with separation in the inertial range of scales of the carrier turbulence in $3 \mathrm{D}$ turbulence and in the inverse cascade of $2 \mathrm{D}$ turbulence, the structure function is known to follow the K41 scaling: 


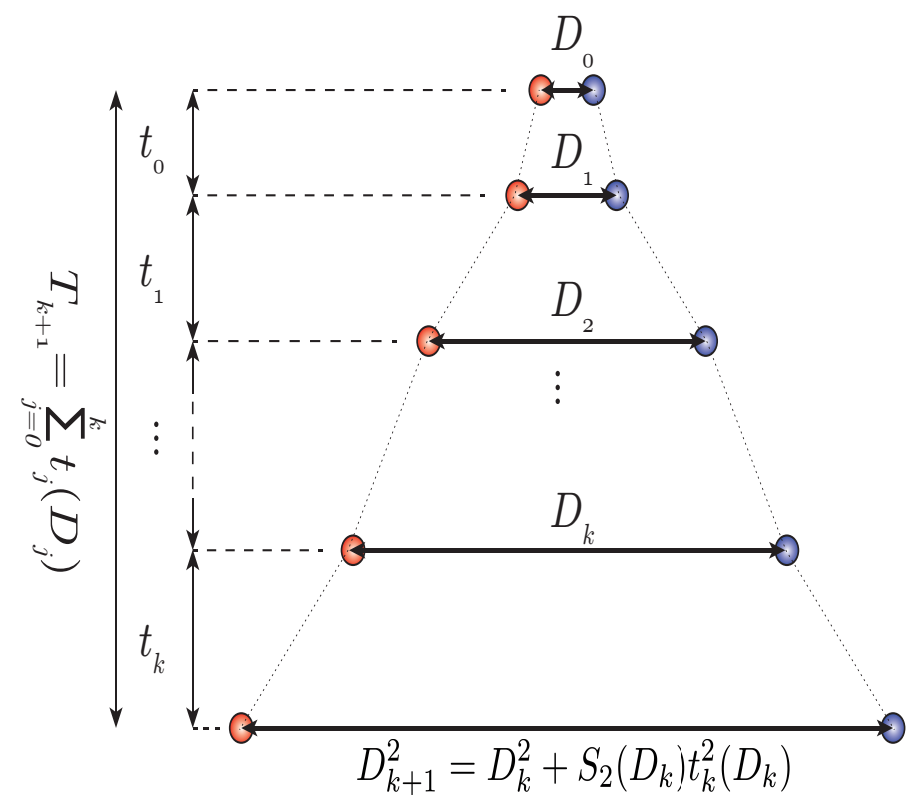

Figure 7. Illustration of the iterative ballistic cascade for the relative separation of particles with initial mean square separation $D_{0}^{2}$ : at each iteration step $k$, the mean square separation between particles grows ballistically from $D_{k}^{2}$ to $D_{k+1}^{2}$ with a growth rate $\sqrt{S_{2}\left(D_{k}\right)}$, during a time lag $t_{k}^{\prime}$. The overall time required to reach separation $D_{k}$ at the iteration number $k$ is $T_{k}=\sum_{j=0}^{k-1} t_{j}^{\prime}\left(D_{j}\right)$.

$$
S_{2}\left(\vec{D}_{0}\right)=C \epsilon^{2 / 3} D_{0}^{2 / 3},
$$

with $D_{0}=\left|\vec{D}_{0}\right|$ and where $C$ is a universal constant. For 3 D-turbulence $C=C^{3 D}=\frac{11}{3} C_{2}^{3 D}$, where $C_{2}^{3 D} \simeq 2.1$ is analytically related to the Kolmogorov constant $C_{K} \simeq C_{2} / 4$ (Sreenivasan, 1995) (characterizing the celebrated $-5 / 3$ spectrum of turbulent kinetic energy $\left(E(k)=C_{K} \epsilon^{2 / 3} k^{-5 / 3}\right)$. We shall therefore refer to $C$ as the Kolmogorov constant in the sequel. The 3D-Kolmogorov constant is then hence $C^{3 D} \simeq \frac{11}{3} \cdot 2.1 \simeq 7.7$ ). The exact same phenomenology can also be applied to the inverse cascade regime of scales of 2D-turbulence, for which $S_{2}$ also obeys K41 scalings, with $C=C^{2 D}=\frac{8}{3} C_{2}^{2 D}$, where the $2 \mathrm{D}$-Kolmogorov constant is $C_{2}^{2 D} \simeq 0.17 C_{K}^{2 D} \simeq 13.2$ (Lindborg (1999)), with $C_{K}^{2 D} \sim 6$ (Boffetta and Ecke (2012)) the spectral 2D-Kolmogorov constant (hence $C^{2 D} \simeq 35.3$.

The iterative ballistic process can now be explicitly written as

$$
D_{k+1}^{2}=D_{k}^{2}+S_{2}\left(D_{k}\right) t_{k}^{\prime 2}\left(D_{k}\right) \quad \text { with }\left\{\begin{array}{l}
S_{2}\left(D_{k}\right)=C \epsilon^{2 / 3} D_{k}^{2 / 3} \\
t_{k}^{\prime}\left(D_{k}\right)=\alpha t_{k}=\alpha S_{2}\left(D_{k}\right) / 2 \epsilon
\end{array},\right.
$$

where the Kolmogorov constant $C$ and the persistence parameter $\alpha$ are the only parameters. The Kolmogorov constant being known, $\alpha$ is the only adjustable parameter of the problem.

The iterative process 9 leads to a simple geometrical progression where

$$
\left\{\begin{array}{c}
D_{k+1}^{2}=A D_{k}^{2} \\
t_{k+1}^{\prime}=A^{1 / 3} t_{k}^{\prime}
\end{array},\right.
$$

with

$$
A=1+\frac{\alpha^{2} C^{3}}{4}
$$

Simple arithmetics then lead to the following relations for the growth of the mean square separation as a function of the total time $T_{k}=\sum_{j=0}^{k-1} t_{j}^{\prime}\left(D_{j}\right)$ :

$$
D_{k}^{2}=g \epsilon\left[T_{k}+\left(\frac{D_{0}^{2}}{g \epsilon}\right)^{1 / 3}\right]^{3}
$$


with

$$
g=\left[2 \frac{A^{1 / 3}-1}{\alpha C}\right]^{3}=\left[2 \frac{\left(1+\frac{\alpha^{2} C^{3}}{4}\right)^{1 / 3}-1}{\alpha C}\right]^{3}
$$

Several points are worth being noted at this point :

- Relation 12 shows that in the long term $\left(T \gg\left(D_{0}^{2} / g \epsilon\right)^{1 / 3}\right)$, the iteration of elementary K41 scale dependent ballistic steps eventually builds a Richardson cubic regime where $D^{2}=g \epsilon T^{3}$.

- It also shows that at intermediate times, the growth follows a similar cubic regime, but with a negative virtual time origin $T_{\text {origin }}=-\left(D_{0}^{2} / g \epsilon\right)^{1 / 3}$ such that $D^{2}=g \epsilon\left(T-T_{\text {origin }}\right)^{3}$, what supports the empirical approach by Ott and Mann (2000) to extract the value of the Richardson constant from short experimental tracks.

- Finally, relation 13 related the Richardson constant to the Kolmogorov constant $C$ and to the persistence parameters.

\subsection{Practical implementation of the ballistic phenomenology}

The practical implementation of the iterative ballistic phenomenlogy only requires the knowledge of the Kolmogorov constant $C$ and the persistence parameter $\alpha$. As discussed in the previous section, the Kolmogorov constant has well accepted values, both for the 3D turbulence case $\left(C^{3 D} \simeq 7.7\right)$ and for the inverse cascade of $\left(2 \mathrm{D}\right.$ turbulence $\left(C^{2 D} \simeq 35.3\right)$. The persistence parameter is a new quantity, which in principle is related to the correlation time of the relative velocity of pairs, which to our knowledge has never been measured yet (neither experimentally nor numerically). We will therefore extract here this parameter by comparing the prediction for the Richardson constant $g$ from eq. 13 to presently well accepted values of $g$.

Figure ??a\&b show the prediction for the Richardon constant as a function of $\alpha$ given by eq. 13 for the $3 \mathrm{D}$ and $2 \mathrm{D}$ turbulence cases. For the 3D case (figure ??a) this is obtained by fixing the value of the Kolmogorov constant to $C^{?} 3 D \simeq 7.7$. By comparing this prediction to the well accepted value for the Richardson constant $g^{3 D} \simeq 0.55$ we can retrieve the optimal value for the persistence parameter $\alpha^{3 D} \simeq 0.12$. Note that $\alpha \simeq 2.8$ would be a priori another possible value of the persistence parameter also consistent with the expected value of the Richardson constant. However, as it will be discussed later, the smallest value of the persistent parameter is to be preferred in order to warrant the validity of the scale-by-scale ballistic approximation.

Figure 9 shows the result of the ballistic phenomenology with $C=C^{3 D}=7.7$ and $\alpha=\alpha^{3 D}=$ 0.12 for the mean square separation as a function of time as given by eq. 12, compared to the direct numerical simulations of 3D homogeneous isotropic turbulence by Bitane et al. (2012). It can be seen that the iterative ballistic phenomenology reproduces almost perfectly the numerical data.

Figure 9 also shows the mean square separation of pairs measured in high resolution particle tracking experiments by (Bourgoin et al., 2006). In those experiments, only the Batchelor ballisitc regime was reported, while no hint of Richardson regime was detected. Figure 9 emphasizes a possible reason for the failure in experiments to observe the Richardson regime : the longest experimental tracks did not exceed a few tenth of $t_{0}$ while the separation needs to be tracked for at least a few $t_{0}$ to reasonably detect the transition toward the cubic regime. A simple possible strategy to improve the chances to observe the cubic regime in experiments would simply consist in better controlling the injection of particle pairs in order to achieve sufficiently small initial separations, hence reducing the time $t_{0}$ required for the transition to occur within experimentally accessible tracking time.

For the 2D turbulence case, eq. 13 can also be used to determine the optimal value of the persistence parameter $\alpha$ compatible with reported values for the forward Richardson constant $g^{2 D}$. Values for $g_{f w d}^{2 D}$ in the literature still span a broad range. Experiments by Jullien et al. (1999) suggest $g_{f w d}^{2 D} \simeq 0.55$, while numerical simulations by Boffetta and Sokolov (2002b) and by Faber and Vassilicos (2009) report $g_{f w d}^{2 D} \simeq 3.8$ and $g_{f w d}^{2 D} \simeq 6.9$ respectively. For the sake of the present discussion, we will consider the value proposed by Faber \& Vassilicos $\left(g^{2 D} \simeq 6.9\right)$ as this study also addresses explicitly and quantitatively the comparison between forward and backward dispersion which will be discussed in section ??. Fig. ??b shows the prediction for the 


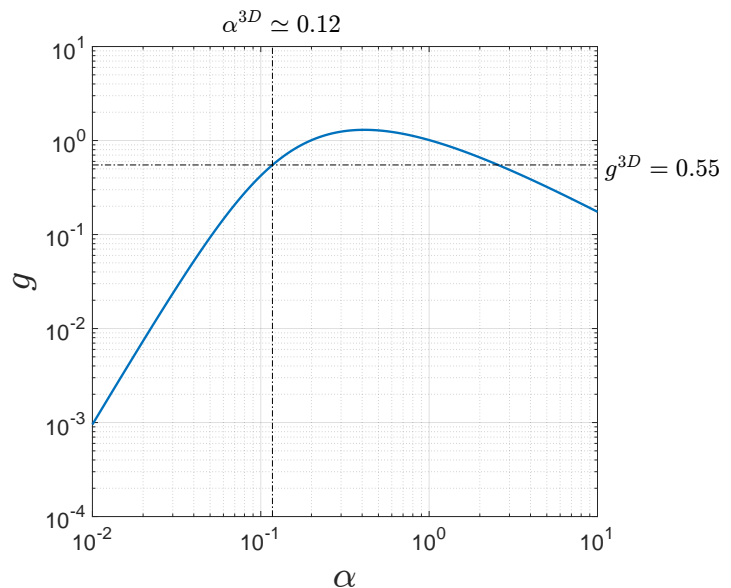

(a)

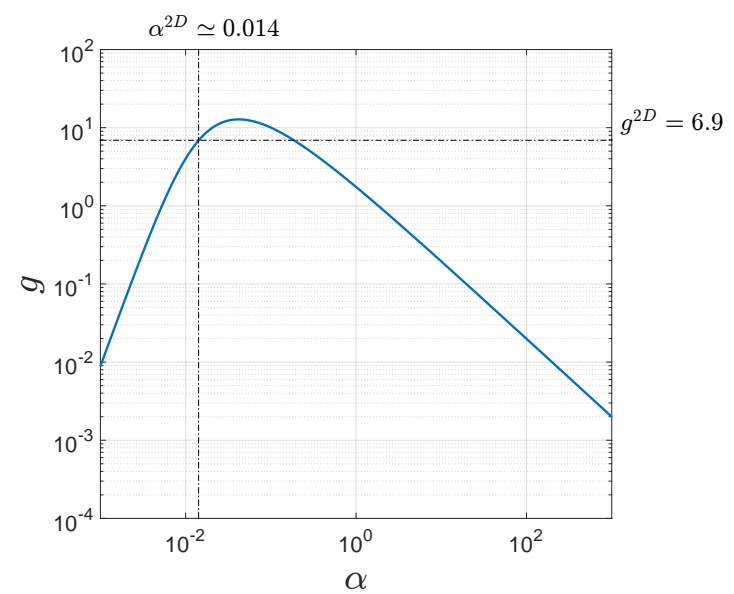

(b)

Figure 8. Dependency of the Richardons constant as a function of the persistence parameter $\alpha$ as predicted by eq. ?? for the 3D case (a), where the Kolmogorov constant has been fixed to $C_{3 D} \simeq 7.7$ anf for the 2D case (b), where the Kolmogorov constant has been fixed to $C_{3 D} \simeq 35.3$. The horizontal dot-dashed lines in each plot indicate the well accepted values for the Richardson constant in both cases and the vertical lines indicate the optimal value of the persistence parameter leading to these values. Note that for the balisitic approximation to be valid, the smallest possible value of $\alpha$ is preferred (see discussion in the text).

Richadson constant as a function of the persistence parameter $\alpha$ for the $2 \mathrm{D}$ case (i.e. from eq. 13 with the Kolmogorov constant fixed to $C_{2 D}=35.3$ ), leading to an optimal value of the persistence parameter of $\alpha^{2 D} \simeq 0.014$.

\subsection{About the time irreversibility of turbulent relative dispersion}

One of the noticeable feature of turbulent dispersion is the temporal asymmetry, meaning that backward and forward dispersion operate at a different rate. The backward dispersion problem is analog to the dispersion problem discussed so far, except that $D_{0}$ is now considered as a final condition rather than a initial condition: considering an ensemble of particles with a given mean square separation $\left\langle D_{0}^{2}\right\rangle$ at $t=0$, what was their mean square separation $\left\langle\left(\vec{D}(-t)-\vec{D}_{0}\right)^{2}\right\rangle$ at earlier times $-t$ ? The concept of backward dispersion is crucial for turbulent mixing and passive scalar studies (Salazar and Collins (2009)) as it determines for instance the rate at which reactants injected at two different source points will eventually get close enough to react. The temporal asymmetry of pair dispersion, was only recently pointed by Sawford et al. (2005) who showed that backward dispersion in Lagrangian stochastic models and in three-dimensional DNS operates at a significantly faster rate compared to forward dispersion. Recent experiments and simulations by Berg et al. (2006) confirm this trend with a ratio of backward to forward Richardson constants of the order of 2 for 3D-turbulence. Insterestingly, numerical simulations of 2D-turbulence in the inverse cascade regime by Faber \& Vassilicos present the opposite asymmetry, 2D forward dispersion operating faster than $2 \mathrm{D}$ backward dispersion. The origin of this asymmetry remained unclear and will be addressed in this section, where an extension of the ballistic phenomenology will be introduced which accounts for time asymetry and for the differences between 2D and 3D turbulence. Sawford et al. (2005) pointed the importance of odd moments of two points velocity statistics for the time irreversibility of relative dispersion while Berg et al. (2006) suggested an explanation based on the strain-tensor eigenvalues, which was however shown by Faber and Vassilicos (2009) to be insufficient to explain the difference between 2D and 3D-turbulence, hence emphasizing the possible role of energy flux accross scales, which goes from large to small scales 
in 3D-turbulence and from small to large scale in 2D-turbulence inverse cascade.

In its present formulation, the iterative ballistic phenomenology is completely time-reversible as the elementary ballistic process in eq. (7) at each scale is quadratic in time and hence fully reversible under the transformation $t \rightarrow-t$. An extension of the iterative ballistic phenomenology to address the question of time assymmetry can be simply introduced by pushing up to third order the Taylor expansion leading to the elementary ballistic process (6). When pushed to third order, the iterative scheme (9), giving the growth of pair separation at iteration between the $k^{t h}$ and the $(k+1)^{t h}$ iteration then becomes :

$$
D_{k+1}^{2}=D_{k}^{2}+S_{2}\left(D_{k}\right) t_{k}^{\prime 2}+S_{a u}\left(D_{k}\right) t_{k}^{\prime 3},
$$

where $S_{a u}(r)=\left\langle\delta_{\vec{r}} \vec{a} \cdot \delta_{\vec{r}} \vec{u}\right\rangle$ is the crossed velocity-acceleration strcture function.

From a physical point of view, the third order term has a clear energetic interpretation. The crossed velocity-acceleration structure function $S_{a u}(r)=\left\langle\delta_{\vec{r}} \vec{a} \cdot \delta_{\vec{r}} \vec{u}\right\rangle$ can indeed be analytically related to the third order Eulerian velocity structure function (and hence to the energy cascade accross scales) directly from Navier-Stokes equation, such that under local stationarity and homogeneity assumptions (see for instance (Mann et al., 1999; Hill, 2006)) :

$$
2\left\langle\delta_{\vec{r}} \vec{a} \cdot \delta_{\vec{r}} \vec{u}\right\rangle=\vec{\nabla} \cdot\left\langle\delta_{\vec{r}} \vec{u} \delta_{\vec{r}} \vec{u} \cdot \delta_{\vec{r}} \vec{u}\right\rangle .
$$

In 3D turbulence, $\vec{\nabla} \cdot\left\langle\delta_{\vec{r}} \vec{u} \delta_{\vec{r}} \vec{u} \cdot \delta_{\vec{r}} \vec{u}\right\rangle=-4 \epsilon$ (what is an exact relation and an alternative version of the Karman-Howarth-Monin relation under local homogeneity and isotropy assumptions Frisch (1995)), what leads to

$$
S_{a u}^{3 D}=-2 \epsilon .
$$

The negative sign in these relation reflects the fact that in 3D turbulence the energy cascade at inertial scales is a direct cascade (energy flows from large to small scales).

Conversely, in the inverse cascade of $2 \mathrm{D}$ turbulence $\vec{\nabla} \cdot\left\langle\delta_{\vec{r}} \vec{u} \delta_{\vec{r}} \vec{u} \cdot \delta_{\vec{r}} \vec{u}\right\rangle=+4 \epsilon(($ Lindborg, 1999)), what leads then to

$$
S_{a u}^{2 D}=+2 \epsilon,
$$

the positive sign refering now to the inverse nature of the energy cadcade.

Note. These relations together with eq. 14 show that the ballistic approximation previously discussed holds as long as $t_{k}^{\prime} \ll\left|S_{2}\left(D_{k}\right) / S_{a u}\left(D_{k}\right)\right|=S_{2}\left(D_{k}\right) / 2 \epsilon$, what according to relation 9 requests $\alpha \ll 1$. This justifies the choice of the smallest relevant persistence parameter.

The crossed velocity-acceleration structure function therefore carries the signature of the energy flux accross scales. In the present context of pair dispersion, the cubic term in eq. (14) should therefore be seen as the Lagrangian signature of the energy flux due to the relative motion (relative velocity and relative acceleration) of particles separating from a given scale to larger scales. The negative sign in the relation $S_{a u}^{3 D}=-2 \epsilon$ in $3 \mathrm{D}$ can be interpreted as the fact that in the forward disperion process, as particles separate (from small to large scales), they climb the energy cascade "upstream", against the energy flux (which flows from large to small scales in the direct 3D cascade), while in the backward case separation pair separation climbs the cascade downstream, with the energy flux. The scenario is reversed in the inverse cascade of $2 \mathrm{D}$ turbulence.

The third order correction to the ballistic process in the extended iterative phenomenology therefore accounts, in the Lagrangian framework of pair dispersion, for the scale asymmetry of energy flux in the turbulent cascade and introduces the notion of Lagrangian temporal asymmetry.

When the relation $S_{a u}^{3 D}=-2 \epsilon$ (for 3D turbulence) is reported in the elementary short term separation process given by eq. 14, we find that for short times the separation is still dominated by the ballistic (quadratic) contribution, with a third order temporal asymmetry such that the difference between short term forward and backward separation is $\left\langle D^{2}\right\rangle(-t)-\left\langle D^{2}\right\rangle(t)=$ $4 \epsilon t^{3}$ (in 3D turbulence). This corresponds to the short term cubic in time asymmetry of relative dispersion recently investigated by Jucha et al. (2014). We will show here that the iterative propagation of this short term asymmetry also builds the long-term asymmetry, in quantitative agreement with previous studies for the Richardson regime asymmetry. Such a connection between 
the short term and long term asymmetry still remained to be established, as pointed by Jucha et al. (2014).

A similar iterative process as the one described previously can indeed be iterated replacing eq. 9 with eq. 14. Considering that $S_{a u}= \pm 2 \epsilon$ (the sign depending on the direction of the turbulent energy cascade), the cubic correction is written as $C_{a u} 2 \epsilon t^{3}$, where $C_{a u}= \pm 1$ depending both on the nature $(2 \mathrm{D}$ vs $3 \mathrm{D})$ of turbulence and on the time direction (forward $v s$ backward disperstion), with the rules:

\begin{tabular}{ccc}
\hline & $3 \mathrm{D}$ & $2 \mathrm{D}$ \\
& (direct cascade) & (inverse cascade) \\
\hline \hline forward & -1 & +1 \\
backward & +1 & -1 \\
\hline
\end{tabular}

The outcome of this calculation leads to an expression for the long term mean square separation growth identical to eq. ??, where the only modification is in the experession for the Richardson constant which now includes the additionnal coefficient $C_{a u}$ accounting for the cubic correction:

$$
g=\left[2 \frac{\left(1+\frac{\alpha^{2} C^{3}}{4}\left(1+C_{a u} \alpha\right)\right)^{1 / 3}-1}{\alpha C}\right]^{3}
$$

As a result, the third order corrected model behaves exactly as the purely ballistic model, except for the relation between the Richardson constant and the model parameters which now includes a corrective term, associated to the asymmetry coefficient $C_{a u}$.

The Richardson constant can the be derived from this relation for the $2 \mathrm{D} / 3 \mathrm{D}$ cases both in the forward/backward dispersion problem, by using the appropriate values of the Kolmogorov and persistence constants already discussed previously (we recall that $C^{3 D} \simeq 7.7, C^{2 D} \simeq 35.3$, $\alpha^{3 D} \simeq 0.14, \alpha^{2 D} \simeq 0.012$ ), and the appropriate values of the asymmetry constant $C_{a u}$ according to the table above. This leads to the following ratios of the forward vs backward Richardson constants:

$$
\frac{g_{b w d}^{3 D}}{g_{f w d}^{3 D}} \simeq 1.9 \quad \text { and } \quad \frac{g_{b w d}^{2 D}}{g_{f w d}^{2 D}} \simeq 0.9
$$

in quantitative agreement with the experiments and simulations by Berg et al. (2006) and with the more recent simulations by Bragg et al. (2014), who all find a ratio $g_{b w d}^{3 D} / g_{f w d}^{3 D} \simeq 2$ for the $3 \mathrm{D}$ turbulence case, and with simulations by Faber and Vassilicos (2009) who report $g_{b w d}^{2 D} / g_{\text {fwd }}^{2 D}=0.92$ for the inverse cascade of $2 \mathrm{D}$ turbulence.

\section{Conclusion}

Anisotropy and two-dimensionalization are two important properties of geophysical flows. The experimental results reported in section 3 show that the small scale local isotropy hypothesis, generally considered in models and simulations might be more controversial than usually believed. Large scale anisotropy appears to persist at small scales, even in the limit of large Reynolds numbers (approaching $R_{\lambda} \sim 10^{3}$ ). The analysis of turbulent relative dispersion reported in sec. 4 show that the rate at which particles separate at inertial scales (an intrinsically Lagrangian problem) can be very simply related to the usual Eulerian phenomenology of turbulence cascade, including the energy spectrum (via the second order Eulerian structure function $S_{2}$ ) and the energy flux (vi the crossed acceleration-velocity structure function, iself related to the third order Eulerian structure function $S_{3}$ ). This allows to simply predict the short and long term time dependencies of the mean square separation by simply as well as subtle effects as temporal asymetry. In particular, the existence of a 2D inverse cascade is responsible for a faster forward dispersion process in 2D while in a direct energy cascade scenario, as in 3D turbulence, backward dispersion operates faster than forward. It could also be that the ballistic iterative process is not limited to the sole case explicitly addressed here (using $S_{2}(r) \propto(\epsilon r)^{2 / 3}$, but can for instance also be applied to predict the dispersion laws for the $2 \mathrm{D}$ direct energy cascade by using the appropriate law $S_{2}(r) \propto \zeta^{2 / 3} r^{2}$, with $\zeta$ the enstrophy dissipation. 


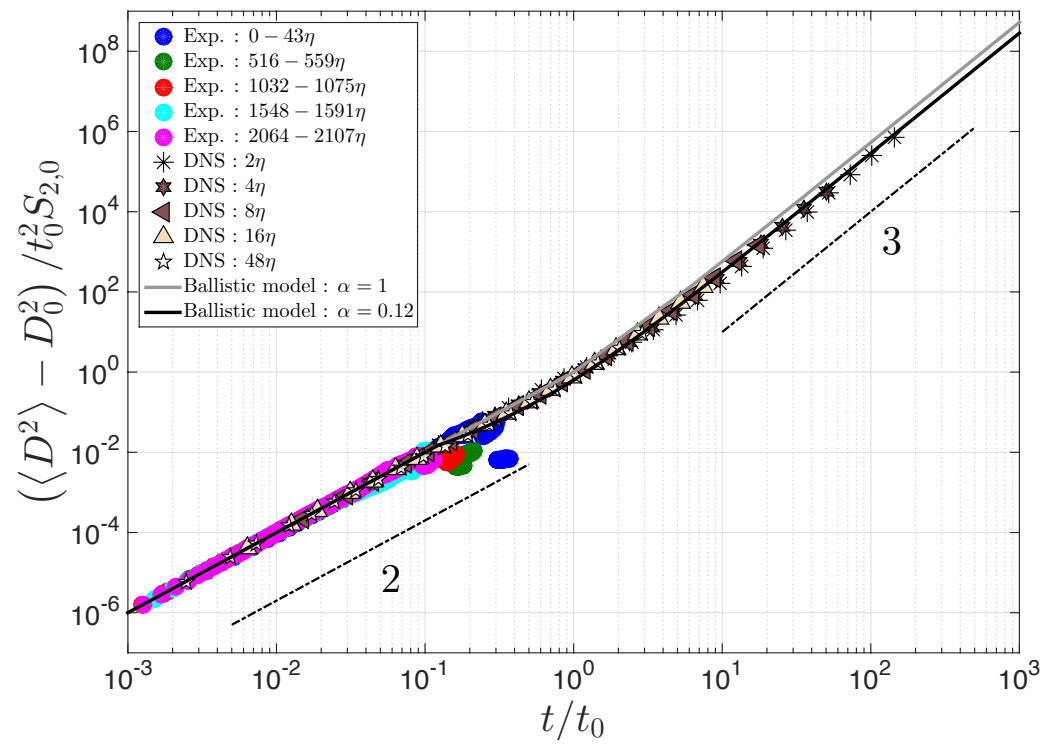

Figure 9. Comparison of the prediction from the ballistic model for two values of the persistence parameter $(\alpha=1$ and $\alpha=0.12)$ and for the mean square separation of particles with different initial separation $D_{0}$ (given in the inset) within inertial scales, obtained in direct numerical simulations (Bitane et al., 2012) and in high resolution particle tracking experiments (Bourgoin et al., 2006). Note that the time axis is normalize by $t_{0}=S_{2}\left(D_{0}\right) / 2 \epsilon$ and the mean square separation is normalized by $t_{0}^{2} S_{2}\left(D_{0}\right)$.

\section{Bibliography}

F Anselmet, Y Gagne, Ej Hopfinger, and R A Antonia. High-order velocity structure functions in turbulent shear flows. Journal of Fluid Mechanics, 140(MAR):63-89, 1984.

A Arneodo, C Baudet, F Belin, R Benzi, B Castaing, B Chabaud, R Chavarria, S Ciliberto, R Camussi, F Chillà, B Dubrulle, Y Gagne, B Hebral, J Herweijer, M Marchand, J Maurer, J. F Muzy, A Naert, A Noullez, J Peinke, F Roux, P Tabeling, W. van de Water, and H Willaime. Structure functions in turbulence, in various flow configurations, at Reynolds number between 30 and 5000, using extended self-similarity. Europhysics Letters (EPL), 34(6):411-416, 1996.

G K Batchelor. The theory of axisymmetric turbulence. Proceedings of The Royal Society of London Series A-Mathematical and Physical Sciences, 186(1007):480-502, 1946.

G K Batchelor. The application of the similarity theory of turbulence to atmospheric diffusion. Quarterly Journal of the Royal Meteorological Sociey, 76(328):133-146, 1950.

Jacob Berg, Beat Lüthi, Jakob Mann, and Søren Ott. Backwards and forwards relative dispersion in turbulent flow: An experimental investigation. Physical Review E, 74(1):016304, 2006.

L Biferale and I Procaccia. Anisotropy in turbulent flows and in turbulent transport. PHYSICS REPORTS-REVIEW SECTION OF PHYSICS LETTERS, 414(2-3):43-164, 2005.

Rehab Bitane, Holger Homann, and Jérémie Bec. Time scales of turbulent relative dispersion. Physical Review E, 86(4):045302, 2012.

G. Boffetta and I. Sokolov. Relative Dispersion in Fully Developed Turbulence: The Richardson's Law and Intermittency Corrections. Physical Review Letters, 88(9):094501, 2002.

G. Boffetta and I. M. Sokolov. Statistics of two-particle dispersion in two-dimensional turbulence. Physics of Fluids, 14(9):3224-3232, 2002.

Guido Boffetta and Robert E. Ecke. Two-Dimensional Turbulence. Annual Review of Fluid Mechanics, 44(1):427-451, 2012.

Mickael Bourgoin. Turbulent pair dispersion as a ballistic cascade phenomenology. Journal of Fluid Mechanics, 772:678-704, 2015.

Mickaël Bourgoin and Haitao Xu. Focus on dynamics of particles in turbulence. New Journal of Physics, 16(8):085010, 2014. 
Mickael Bourgoin, Nicholas T Ouellette, Haitao Xu, Jacob Berg, and Eberhard Bodenschatz. The role of pair dispersion in turbulent flow. Science, 311(5762):835-838, 2006.

Mickael Bourgoin, Jean-François Pinton, and Romain Volk. Lagrangian methods in experimental fluid mechanics. In Thomas von Larcher and Paul D. Williams, editors, Modeling Atmospheric and Oceanic Flows: Insights from Laboratory Experiments and Numerical Simulations, page 360. John Wiley \& Sons, Inc., 2014. ISBN 1118855930. URL https : //books . google. com/books?hl=fr\&lr=\&id=8GezBQAAQBAJ\&pgis=1.

Andrew D. Bragg, Peter J. Ireland, and Lance R. Collins. Forward and backward in time dispersion of fluid and inertial particles in isotropic turbulence. arXiv:1403.5502 [physics.flu-dyn], page 30, 2014.

S Chandrasekhar. The theory of axisymmetric turbulence. Philosophical Transactions of The Royal Society of London Series A-Mathematical and Physical Sciences, 242(855):557-577, 1950.

S Y Chen, B Dhruva, S Kurien, K R Sreenivasan, and M A Taylor. Anomalous scaling of low-order structure functions of turbulent velocity. Journal Of Fluid Mechanics, 533:183-192, 2005.

M Chertkov, A Pumir, and B I Shraiman. Lagrangian tetrad dynamics and the phenomenology of turbulence. Physics of Fluids, 11(8):2394-2410, 1999.

P A Davidson and B R Pearson. Identifying Turbulent Energy Distributions in Real, Rather than Fourier, Space. Phys. Rev. Lett., 95(21):214501, 2005.

T. Faber and J. C. Vassilicos. Turbulent pair separation due to multiscale stagnation point structure and its time asymmetry in two-dimensional turbulence. Physics of Fluids, 21(1):015106, 2009.

G Falkovich, K Gawedzki, and M Vergassola. Particles and fields in fluid turbulence. Reviews of Modern Physics, 73(4):913-975, 2001.

U Frisch. Turbulence: The Legacy of A. N. Kolmogorov. Cambridge University Press, Cambridge, England, 1995.

S Garg and Z Warhaft. On the small scale structure of simple shear flow. PHYSICS OF FLUIDS, 10(3):662-673, 1998.

S R HANNA. Lagrangian and Eulerian time-scale relations in the daytime boundary-layer. Journal Of Applied Meteorology, 20(3):242-249, 1981.

RJ Hill. Opportunities for use of exact statistical equations. Journal of Turbulence, 7:43, 2006.

J Hinze. Turbulence. McGraw-Hill, 1959.

Jennifer Jucha, Haitao Xu, Alain Pumir, and Eberhard Bodenschatz. Time-reversal-symmetry Breaking in Turbulence. Physical Review Letters, 113(5):054501, 2014.

Marie-caroline Jullien, Jérôme Paret, and Patrick Tabeling. Richardson Pair Dispersion in TwoDimensional Turbulence. Physical Review Letters, 82(14):2872-2875, 1999.

A. Kolmogorov. The local structure of turbulence in incompressible viscous fluid for very large Reynolds numbers. Dokl. Akacl. Nauk SSSR, 30:301-305, 1941.

A N Kolmogorov. Refining the Notions of the Local Structure of Turbulence in an Incompressible Viscous Fluid at High Reynolds Numbers. In Mecanique de la turbulence, pages 447-458. CNRS, Paris, 1962.

R C Lien and E A D'Asaro. The Kolmogorov constant for the Lagrangian velocity spectrum and structure function. Physics Of Fluids, 14(12):4456-4459, 2002.

R-C. Lien, E A D'Asaro, and G T Dairiki. Lagrangian frequency spectra of vertical velocity and vorticity in high-Reynolds-number oceanic turbulence. Journal of Fluid Mechanics, 362:177, 1998.

Erik Lindborg. Can the atmospheric kinetic energy spectrum be explained by two-dimensional turbulence? Journal of Fluid Mechanics, 388:259-288, 1999.

J Mann, S Ott, and J S Andersen. Experimental study of relative, turbulent diffusion. Technical Report Riso-R-1036 (EN), Risoe National Laboratory, Roskilde, Denmark, 1999.

Nicolas Mordant, Pascal Metz, Olivier Michel, and J.-F. Jean-François Pinton. Measurement of Lagrangian Velocity in Fully Developed Turbulence. Physical Review Letters, 87(21):214501, 2001.

A M Oboukhov. On the Distribution of Energy in the Spectrum of a Turbulent Flow. Izv. Akad. Nauk SSSR, 5:453-466, 1941.

A M Obukhov. Some Specific Features of Atmospheric Turbulence. Journal of Fluid Mechanics, 13:77-81, 1962.

Soren Ott and Jakob Mann. An experimental investigation of the relative diffusion of particle pairs in three-dimensional turbulent flow. Journal of Fluid Mechanics, 422:207-223, 2000. 
Nicholas T Ouellette, Haitao Xu, Mickaël Bourgoin, and Eberhard Bodenschatz. Small-scale anisotropy in Lagrangian turbulence. New Journal of Physics, 8(6):102, 2006.

M Ould-Rouiss. The axisymmetric equivalent of Kolmogorov's equation. European Physical Journal B, 23(1):107-120, 2001.

G Parisi and U Frisch. A multifractal model of intermittency. In M.Ghil, R Benzi, and G Parisi, editors, Turbulence and predictability in geophysical fluid dynamics and climate dynamics, pages 84-87, Amsterdam, 1985. Eds. North Holland.

Alain Pumir and Boris I Shraiman. Persistent Small Scale Anisotropy in Homogeneous Shear Flows. Phys. Rev. Lett., 75(17):3114-3117, 1995.

Lewis F Richardson. Atmospheric Diffusion Shown on a Distance-Neighbour Graph. Proceedings of the Royal Society of London, Series A, 110(756):709-737, 1926.

H C RODEAN. The universal constant for the lagrangian structure-function. Physics of Fluids A, 3(6):1479-1480, 1991.

Juan P L C Salazar and Lance R Collins. Two-Particle Dispersion in Isotropic Turbulent Flows. ANNUAL REVIEW OF FLUID MECHANICS, 41:405-432, 2009.

B L Sawford. Reynolds number effects in Lagrangian stochastic models of tur bulent dispersion. Physics of Fluids A, 3(6):1577-1586, 1991.

Brian Sawford. Turbulent Relative Dispersion. Annual Review of Fluid Mechanics, 33:289-317, 2001.

Brian L. Sawford, P. K. Yeung, and Michael S. Borgas. Comparison of backwards and forwards relative dispersion in turbulence. Physics of Fluids, 17(9):095109, 2005.

Katepalli R Sreenivasan. On the universality of the Kolmogorov constant. Physics of Fluids, 7(3): 2778-2784, 1995.

G I Taylor. Diffusion by continuous movements. Proc. Lond. Math. Soc., 20:196-212, 1922.

$\mathrm{H}$ Tennekes. Eulerian and Lagrangian time microscales in isotropic turbulence. Journal of Fluid Mechanics, 67(3):561-567, 1975.

K A Weinman and A Y Klimenko. Estimation of the Kolmogorov constant C-0 by direct numerical simulation of a continuous scalar. Physics of Fluids, 12(12):3205-3220, 2000.

$\mathrm{H} \mathrm{Xu}$, Mickael Bourgoin, N T Ouellette, and E Bodenschatz. High order Lagrangian velocity statistics in turbulence. Physical Review Letters, 96(2), 2006.

P K Yeung. Lagrangian investigations of turbulence. Annual Review of Fluid Mechanics, 34: $115-142,2002$. 